\title{
Functional implications of K63-linked ubiquitination in the iron deficiency response of Arabidopsis roots
}

\author{
I-Chun Pan ${ }^{1}$ and Wolfgang Schmidt ${ }^{1,2,3}$ * \\ 1 Institute of Plant and Microbial Biology, Academia Sinica, Taipei, Taiwan \\ ${ }^{2}$ Biotechnology Center, National Chung-Hsing University, Taichung, Taiwan \\ ${ }^{3}$ Genome and Systems Biology Degree Program, College of Life Science, National Taiwan University, Taipei, Taiwan
}

\section{Edited by:}

Pascal Genschik, Centre National de la Recherche Scientifique, France

\section{Reviewed by:}

Karel Riha, Gregor Mendel Institute,

Austria

Xun Xu, Beijing Genomics

Institute-Shenzhen, China

\section{*Correspondence:}

Wolfgang Schmidt, Institute of Plant and Microbial Biology, Academia

Sinica, Academia Road 128,

Taipei 11529, Taiwan

e-mail:wosh@gate.sinica.edu.tw
Iron is an essential micronutrient that plays important roles as a redox cofactor in a variety of processes, many of which are related to DNA metabolism. The E2 ubiquitin conjugase UBC13, the only E2 protein that is capable of catalyzing the formation of non-canonical K63-linked ubiquitin chains, has been associated with the DNA damage tolerance pathway in eukaryotes, critical for maintenance of genome stability and integrity. We previously showed that UBC13 and an interacting E3 ubiquitin ligase, RGLG, affect the differentiation of root epidermal cells in Arabidopsis. When grown on iron-free media, Arabidopsis plants develops root hairs that are branched at their base, a response that has been interpreted as an adaption to reduced iron availability. Mutations in UBC13A abolished the branched root hair phenotype. Unexpectedly, mutations in RGLG genes caused constitutive root hair branching. Based on recent results that link endocytotic turnover of plasma membranebound PIN transporters to K63-linked ubiquitination, we reinterpreted our results in a context that classifies the root hair phenotype of iron-deficient plants as a consequence of altered auxin distribution. We show here that UBC13A/B and RGLG1/2 are involved in DNA damage repair and hypothesize that UBC13 protein becomes limited under iron-deficient conditions to prioritize DNA metabolism. The data suggest that genes involved in combating detrimental effects on genome stability may represent essential components in the plant's stress response.

Keywords: auxin, DNA repair, endocytosis, iron deficiency, K63-linked polyubiquitylation, root hairs

\section{INTRODUCTION}

Iron participates in a variety of vital processes as a redox cofactor and is an indispensable element for virtually all organisms. In plants, suboptimal iron availability results in decreased yield and reduced quality of edible plant parts, causing severe economic losses. When plants are the major source of dietary iron, a low iron concentration may pose severe health problems. Iron deficiency-induced anemia (IDA) is the most widespread nutritional disorder worldwide. In severe cases, IDA can affect infant development and increase the risk of maternal and child mortality. Due to the limited solubility of iron in aerated soils, mechanisms have evolved that aid in mobilizing otherwise sparingly soluble $\mathrm{Fe}$ (III)oxihydroxides, which represent the prevailing form of iron in most soils at neutral or basic $\mathrm{pH}$. Induction of proton-translocating P-type ATPases decreases the rhizospheric $\mathrm{pH}$, thereby increasing the activity of $\mathrm{Fe}^{3+}$ by a factor of 1,000 for each unit the $\mathrm{pH}$ decreases (Santi et al., 2005; Santi and Schmidt, 2008, 2009). In addition, secretion of iron binding compounds (IBCs) facilitates the mobilization of iron particularly at high $\mathrm{pH}$ (Susín et al., 1993; Jin et al., 2007; Fourcroy et al., 2013; RodríguezCelma et al., 2013). A further set of supposedly separately regulated responses comprise morphological changes such as the formation of additional root hairs and invaginations of secondary walls in the rhizodermis/hypodermis, responses that were suggested to improve iron acquisition by increasing the absorptive surface area
(Schikora and Schmidt, 2001; Schmidt et al., 2003). In contrast to phosphate deficiency, which triggers a nutrient-specific and conserved set of developmental responses including denser and longer root hairs (Ma et al., 2001; Savage et al., 2013) and a dramatically altered root architecture where primary root growth is attenuated and lateral root formation is stimulated (Ticconi et al., 2009), the morphological responses to iron deficiency are less well studied and appear to be less conserved among species. While some plants such as sunflower and cucumber produce very dense root hairs in response to iron deficiency (Landsberg, 1996; Li and Schmidt, 2010), other species such as Plantago lanceolata and tomato show only a moderate increase in root hair frequency (Schmidt and Bartels, 1996; Schikora and Schmidt, 2002). Interestingly, both the induction of cell wall invaginations and the formation of extra root hairs are inducible by application of exogenous auxin, suggesting to us that auxin is involved in the induction of these responses (Landsberg, 1996; Schmidt et al., 2003).

In Arabidopsis, the morphological responses to iron deficiency are neither pronounced nor uniformly described. Branching of the hairs was described as a major response and interpreted as an alternative to longer and/or denser root hairs as a strategy to increase the surface area of the roots (Müller and Schmidt, 2004). Instead, formation of shorter and misshapen root hairs was described for iron-deficient Arabidopsis plants by Dinneny et al. 
(2008), indicating that subtle differences in media composition and growth conditions may impact the phenotypic readout.

While the physiological responses to iron deficiency are well explored at the molecular level, not much information is available regarding the mechanisms controlling the morphological alterations typical of iron-deficient plants. So far, the only gene with a putative function in iron deficiency-induced formation of root hairs is the ubiquitin conjugase UBC13. UBC13 was identified in the root hair zone of iron-deficient cucumber plants by a proteomic approach and cloned using the CODEHOP strategy (Li and Schmidt, 2010). The sequence of UBC13 is highly conserved among eukaryotes, and its function has been related to the error tolerance branch of DNA repair in yeast and Arabidopsis and to the NF- $\mathrm{B}$ signal transduction pathway in mammals (Hofmann and Pickart, 1999; Wen et al., 2006; Wu et al., 2009). In Arabidopsis, UBC13 is encoded by two close sequelogs, $U B C 13 A$ (UBC35) and $U B C 13 B$ (UBC36). UBC13 is the only known E2 ubiquitin conjugating enzyme that is capable of catalyzing the formation of ubiquitin chains linked to K63, a function that appears to be conserved in eukaryotes (Hofmann and Pickart, 1999). In contrast to the formation canonical of ubiquitin chains linked via K48, which target proteins for degradation via the $26 \mathrm{~S}$ proteasome, proteins conjugated to K63-linked polyubiquitin chains are involved in signaling and in the coordination of cellular processes such as endocytotic trafficking and DNA repair. UBC13 acts in conjunction with E3 ligases such as the RING domain ligase protein RGLG2 that has been shown to interact with UBC13 in Arabidopsis (Yin et al., 2007). Non-canonical ubiquitation through K63-linked ubiquitin chains is required for DNA damage tolerance, a pathway that allows the bypass of lesions in the DNA template during replication. The AtUBC13 genes complemented the yeast $u b c 13$ null mutant for sensitivity to DNA damaging agents and for spontaneous mutagenesis, suggesting that in Arabidopsis UBC13 proteins are involved in the error-free DNA damage tolerance pathway (Wen etal., 2006). The UBC enzyme variant Mms2 is an E2-like protein that interacts with Ubc13 in eukaryotic cells (ubiquitin conjugating enzyme variant, UEV; Hofmann and Pickart, 1999; VanDemark et al., 2001). The Arabidopsis genome harbors four Mms2 homologs, UEV1A to UEV1D, all of which can form stable complexes with UBC13 from yeast and Arabidopsis (Wen etal., 2008). For AtUEV1D a function in DNA damage response has been experimentally verified (Wen etal., 2008). We here present evidence that both UBC13 and RGLG1/2 are critical in DNA damage repair and hypothesize that the root hair phenotype of iron-deficient plants is caused by diminished availability of RGLG1/2 protein, probably due to stress-induced movement from the plasma membrane to the nucleus and subsequently changed auxin distribution in epidermal cells. We further speculate that under iron-deficient conditions different E2-E3 complexes are favored in the nucleus to prioritize genome stability.

THE Arabidopsis IRON DEFICIENCY ROOT HAIR PHENOTYPE: A CONSEOUENCE OF CHANGES IN AUXIN DISTRIBUTION?

The branched root hair phenotype of iron-deficient plants differs from that of phosphate-deficient plants, which form longer and denser root hairs resulting from restricted longitudinal elongation of root epidermal cells and additional cell fate assignment by increased expression of the Myb-type transcription factor ETC1 (Ma et al., 2001; Müller and Schmidt, 2004; Savage et al., 2013). Branched root hairs have also been described for auxin-related mutants such as axr1, aux1, and axr2, and for mutants defective in actin filament organization or vesicle transport such as scn1 or tip1 (see Guimil and Dunand, 2007 for an overview). Plants harboring mutations in $U B C 13 A$ do not respond to iron deficiency with the formation of branched root hairs. Moreover, $u b c 13 a u b c 13 b$ double mutants have shorter root hairs compared with the wild type, a trait that was also observed in some auxindeficient mutants such as trh1 (Vicente-Agullo et al., 2004; Li and Schmidt, 2010). Transgenic plants overexpressing UBC13A (UBC13A OE) showed a phenotype that was essentially similar to that of the wild type when grown on iron-free media, with no further increase in the number of branched hairs. Iron-sufficient UBC13A OE plants are indistinguishable from Col-0 plants, indicating that an iron deficiency signal is required for the induction of root hair branching. Notably, double mutants defective in the expression of the E3 ligases RGLG1 and RGLG2 constitutively displayed the branched root hair phenotype (Li and Schmidt, 2010). This was an unexpected result since disruption of the E2/E3 cascade should result in similar phenotypes, regardless of the site of disruption.

Interestingly, the phenotype of rglg1 rglg2 mutants could be rescued by omitting phosphate from the growth media ( $\mathrm{Li}$ and Schmidt, 2010). Such a rescue by phosphate deficiency was described for the short root hair phenotype of the auxin transport mutant trh1 (Müller and Schmidt, 2004). TRH1 is a member of the AtKT/AtKUP/AtHAK family of potassium carriers required for the correct distribution of auxin (Vicente-Agullo et al., 2004). It seems reasonable to speculate that altered auxin metabolism is the cause of the variable phenotype of the mutants grown under the different conditions. In support of this assumption, DR5-GUS reporter lines showed reduced GUS expression under iron-deficient conditions (Lan et al., 2012), indicative of decreased levels of or a diminished responsiveness to auxin. In phosphatedeficient DR5-GUS plants, staining was more intense than under control conditions, indicating increased auxin levels in this growth type. Together these results suggest that compromised auxin transport or metabolism could be the cause for the branched root hair phenotype under iron-deficient conditions.

\section{A MODEL FOR THE FUNCTION OF K63-LINKED UBIOUITINATION IN ROOT HAIR CELLS}

The following model would explain the results obtained by us ( $\mathrm{Li}$ and Schmidt, 2010) and others (Figure 1). The model is based on observations made in Arabidopsis but may also apply to other species. Under iron-sufficient conditions, RGLG1/2 binds a protein $\mathrm{X}$ that, when not captured by RGLG1/2, acts as an inhibitor of proper root hair initiation, probably via a reduction of auxin responsiveness. Protein X may act directly or indirectly on auxin distribution. AtUBC13 is not induced by iron deficiency (Li and Schmidt, 2010) and not much affected by other stresses ${ }^{1}$ and thus

${ }^{1}$ https://www.genevestigator.com/ 


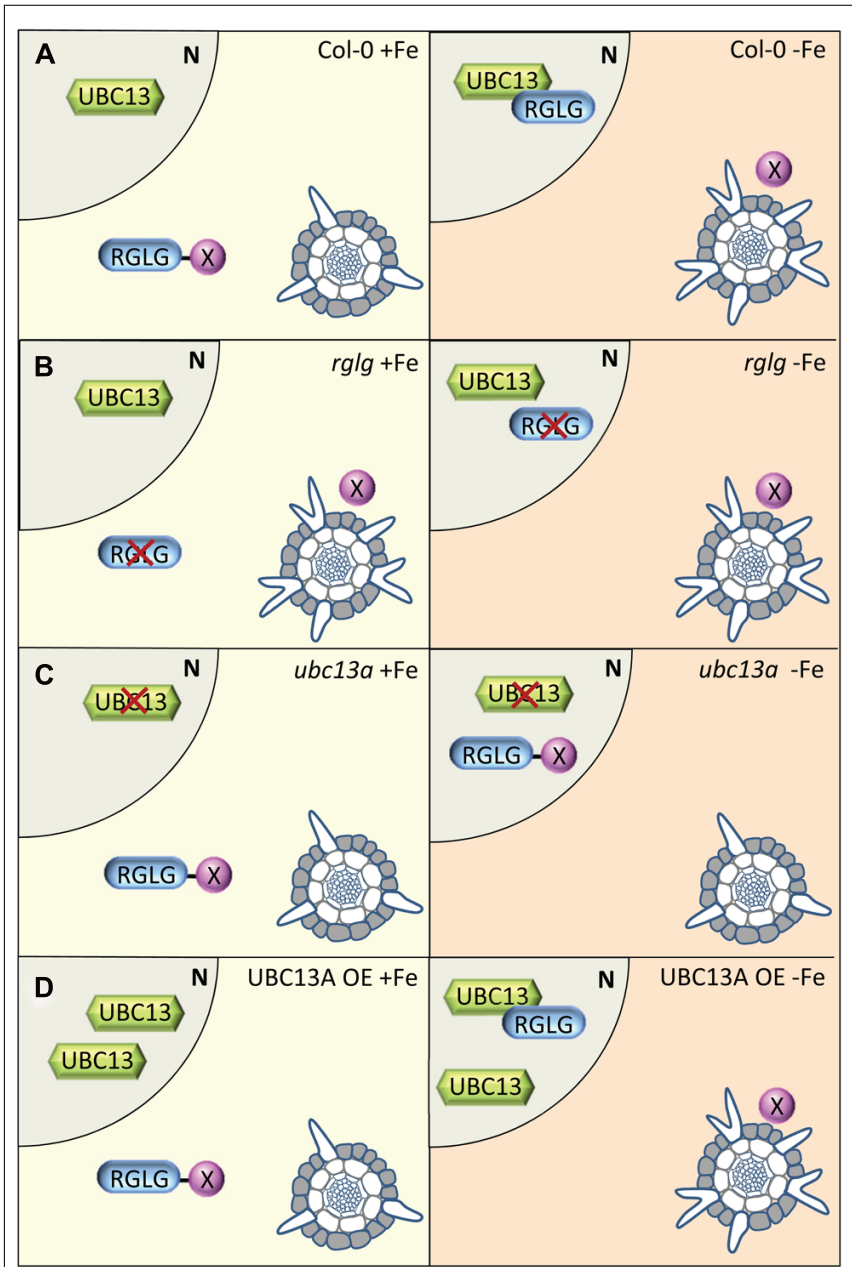

FIGURE 1 | Model depicting putative interactions of UBC13 and RGLG proteins and their effects on root hair differentiation. (A) In wild-type roots, protein $\mathrm{X}$ is bound to RGLG proteins, preventing the formation of branched root hairs. UBC13 is present, but only under iron-deficient conditions UBC13 interacts with RGLG proteins which move to the nucleus. The interaction of $U B C 13$ with $R G L G 1 / 2$ releases protein $X$ which leads to the formation of branched hairs. (B) In rglg mutants, protein $X$ is free both in the presence and absence of iron, causing root hairs to branch independent on the iron supply. (C) In root hair cells of $u b c 13$ plants, no interaction between UBC13 and RGLG1/2 can occur, leaving protein $X$ inactive under both iron-sufficient and iron-deficient conditions. (D) Overexpression of UBC13 increases the level of UBC13 protein but does not affect UBC13-RGLG1/2 interaction, resulting in a phenotype similar to that of the wild type.

likely to be present in iron-sufficient plants in equal amounts. In iron-deficient roots, RGLG1/2 is located to the nucleus where it interacts with UBC13. The UBC13-RGLG1/2 complex formation releases protein $\mathrm{X}$ from RGLG, resulting in a decreased auxin concentration (Figure 1A). Stress-induced movement of RGLG2 from the plasma membrane to the nucleus has been shown previously (Cheng et al., 2012). In rglg1 rglg2 mutants, no UBC13-RGLG1/2 interaction is possible, making protein $\mathrm{X}$ available both under control and iron-deficient condition. Although K63-linked ubiquitin chain-supporting enzymes such as UBC13 are present in irondeficient rglg1 rglg2 plants, they would not affect the abundance of protein $\mathrm{X}$ and cause a constitutive branching root hair phenotype (Figure 1B). In $u b c 13 a$ plants, branched root hairs are neither formed under iron-sufficient nor under iron-deficient conditions. Adopting the scenario outlined above, protein $\mathrm{X}$ remains tightly bound to RGLG1/2, keeping auxin levels (or responsiveness) up and preventing the formation of branched hairs (Figure 1C). Over-abundance of UBC13 in UBC13 OE lines is not affecting root hair branching under control conditions (Figure 1D). Under iron-deficient conditions, RGLG1/2 moves to the nucleus, releases protein $\mathrm{X}$ resulting in a phenotype that is undistinguishable from the wild type.

We can only speculate on the nature of protein X. A possible scenario implies that protein $\mathrm{X}$ post-translationally regulates auxin distribution or metabolism. The plasma membrane-localized auxin carrier protein PIN2 is constitutively recycled by endocytosis (Benjamins and Scheres, 2008; Grunewald and Friml, 2010). Recently, RGLG proteins were shown to be involved in the control of the proteolytic turnover of PIN2 via K63-linked ubiquitination (Leitner et al., 2012). Loss of PIN2 ubiquitination interferes with vacuolar targeting, stabilizes PIN2, and alters auxin availability in Arabidopsis roots. rglg1 rglg2 mutants had reduced auxin levels and transgenic rglg1 rglg2 plants carrying the DR5-reporter construct showed reduced auxin responsiveness (Yin et al., 2007). Translated to the model, PIN2 could represent protein X. A possible scenario is outlined in Figure 2. Under iron-sufficient conditions, RGLG proteins mediates K63linked ubiquitination of PIN2, thereby controlling its proteolytic turnover (Figure 2A). Loss of PIN2 polyubiquitination, caused for example by a mutation in $R G L G 1 / 2$, causes an arrest of endocytotic cycling of PIN2, decreased auxin levels and, ultimately, branching of root hairs. Such a scenario may apply to iron-deficient plants in which UBC13 is recruited by RGLG1/2 in the nucleus, compromising the ubiquitination of PIN2, which accumulates in the cell (Figure 2B). Recruitment of UBC13 may be facilitated by translocation of RGLG from the plasma membrane to the nucleus.

UBC13 was suggested to be involved in the error-free DNA damage repair pathway (Wen et al., 2006). In yeast, this function is fulfilled by an Ubc13-Mms2-Rad5-mediated polyubiquitination of the homotrimeric protein complex proliferating cell nuclear antigen (PCNA); in humans, PCNA ubiquitination is catalyzed by the Rad5 homologs HLTF and SHPRH (Ulrich, 2007; Chang and Cimprich, 2009). A role for AtRAD5A in DNA repair has been demonstrated (Chen et al., 2008; Mannuss et al., 2010; Wang etal., 2011) and an involvement of UBC13-UEV1 and AtRAD5A in this pathway in Arabidopsis represents a likely scenario.

In $u b c 13 a$ mutant plants, several iron-responsive genes are less induced than in the wild type ( $\mathrm{Li}$ and Schmidt, 2010), indicative of an involvement of UBC13A in processes that utilizes iron. For example, induction of the transcription factor $b H L H 38$, an essential regulator of the iron deficiency response, was markedly less pronounced in roots of the $u b c 13 a$ mutant. Notably, under irondeficient conditions the expression of two genes encoding the iron storage proteins FER 1 and FER2 was higher in $u b c 13 a$ plants when compared with the wild type, suggesting a higher iron status of the mutant. These results may be interpreted in a sense that more iron 


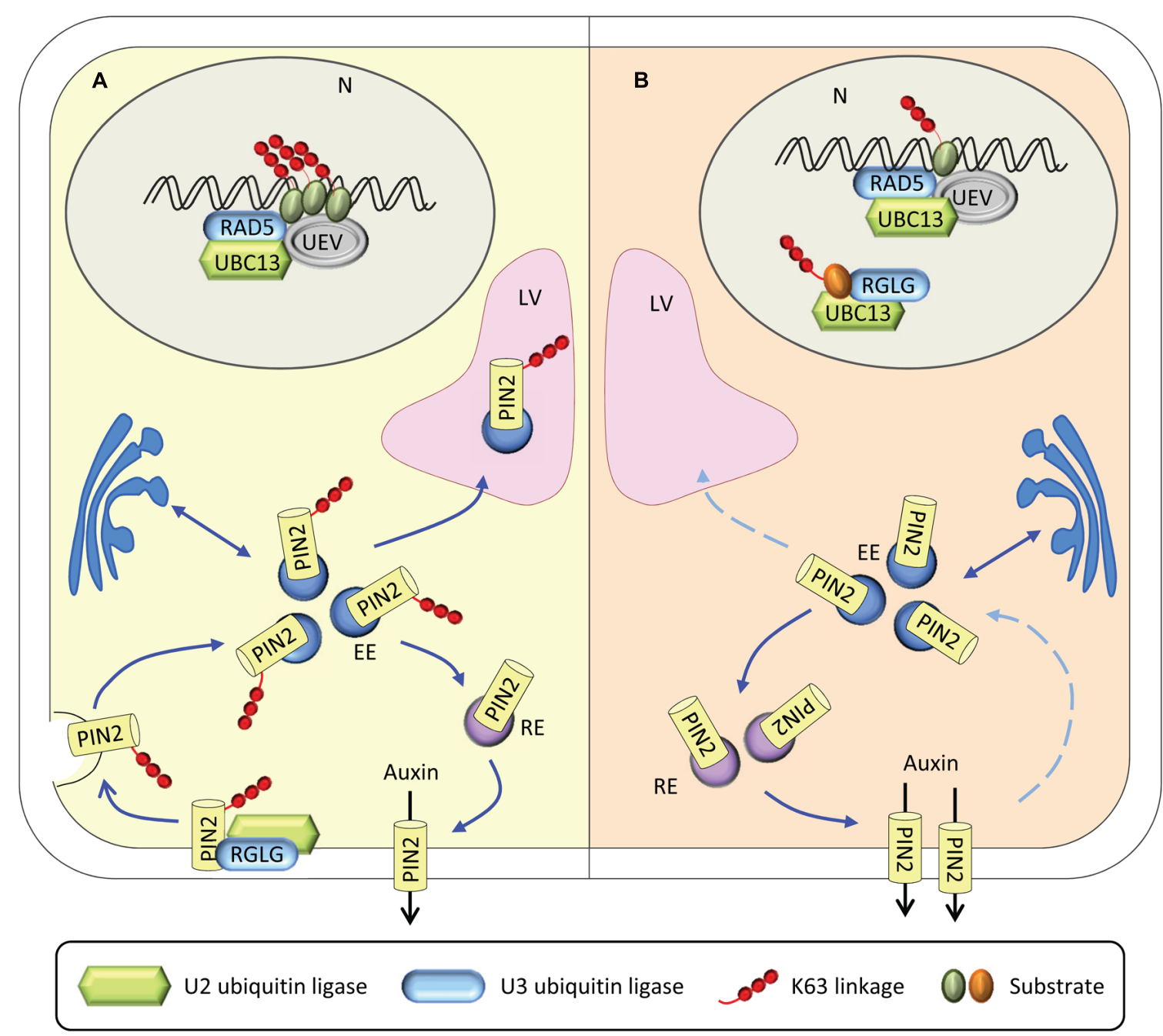

FIGURE 2 | Experimental evidence for processes associated with the formation of K63-linked ubiquitin chains. (A) Under control conditions, RGLG mediates the endocytic turnover of PIN2, thereby regulating the distribution of auxin. UBC13-UEV1-RAD5 is involved in housekeeping DNA damage repair. (B) Under iron-deficient conditions, RGLG moves to the nucleus where it interacts with UBC13. UBC13-RGLG1/2 might be associated with DNA repair or with other processes related to genome stability. Loss of PIN2 ubiquitination cause a cessation of endocytotic PIN2 cycling, thereby altering auxin distribution. N, nucleus; LV, Iytic vacuole; EE early endosome; $R E$, recycling endosome. is available if the UBC13-RGLG pathway is not engaged. Based on the conserved function of UBC13 in DNA damage repair among eukaryotes, it can be assumed that the UBC13-RGLG1/2 complex participates in DNA damage repair.

To test our hypothesis, we germinated seeds from wild-type plants, $r g \lg 1 \mathrm{rglg} 2$, and $u b c 13 a u b c 13 b$ double mutants on media containing various concentrations of the DNA-damaging agent methyl methanesulfonate (MMS). While wild-type plants were largely unaffected both under iron-sufficient and iron-deficient conditions, the germination rate of $u b c 13 a u b c 13 b$ double mutants showed a dramatic decrease in germination rate which was more pronounced under iron-deficient conditions (Figure 3). rglg1 rglg2 mutants showed a less pronounced and iron-independent decrease in seed germination (Figure 3). These data are consistent with the assumption that under iron-deficient conditions RGLG1/2 is translocated into the nucleus where it may associate with UBC13 and other E2 proteins, thereby diminishing the efficiency of UBC13 in DNA damage tolerance. UBC13 may have critical, currently unknown functions in DNA metabolism in conjunction with RGLG1/2, probably associated with genome stability (Figure 2B).

\section{THE ROLE OF IRON IN DNA SYNTHESIS AND REPAIR}

Iron is required for several DNA-related processes. For example, ribonucleotide reductases (RNRs) require iron as an essential cofactor and iron deficiency leads to reduced RNR activity (Cavanaugh et al., 1985; Saletta et al., 2011). RNRs activity is linked to DNA synthesis and tightly regulated in response to iron deficiency in yeast and mammals to assure accurate DNA replication (Furukawa et al., 1992). In yeast, a post-transcriptional regulatory mechanism promotes destabilization of mRNAs involved in non-essential pathways and degradation of such transcripts via 


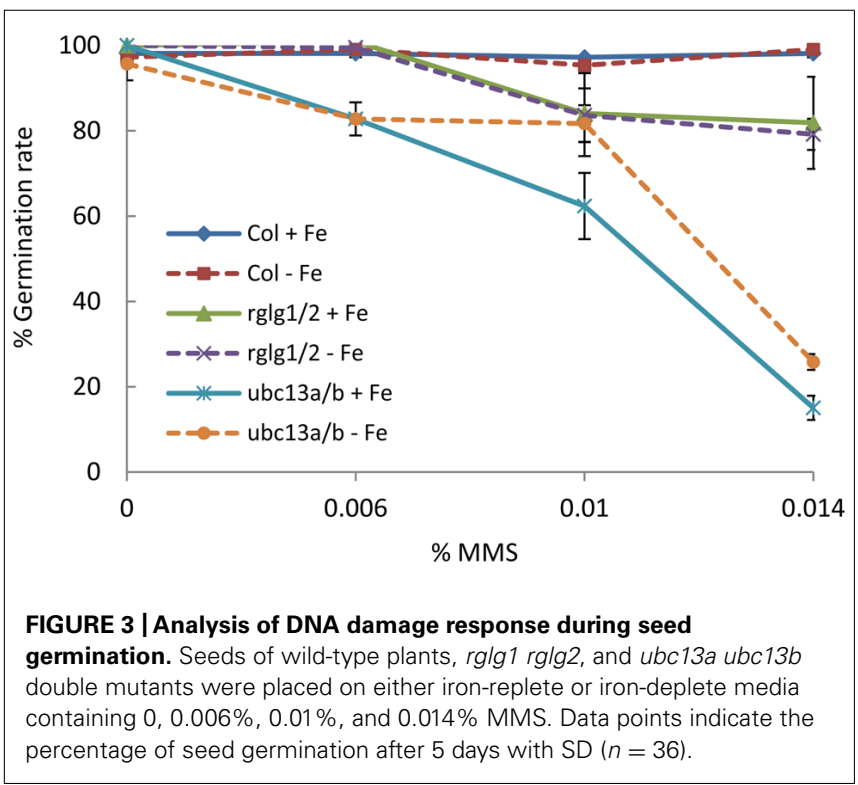

AU-rich element (ARE)-mediated decay (AMD), and supports DNA synthesis and repair via activation of RNR by the Cth2 protein (Martínez-Pastor et al., 2013). Interestingly, many of the down-regulated transcripts are related to the tricarboxylic acid cycle or participate in mitochondrial electron transport, switching respiration to fermentative metabolism (Sanvisens et al., 2011), a response that has also been described for iron-deficient Arabidopsis roots (Thimm et al., 2001). Also, storage of iron in the vacuole via CCC1 is inhibited by this pathway, a further parallel to Arabidopsis roots in which CCC1-like vacuolar iron transporters are down-regulated in response to iron deficiency (Gollhofer et al., 2011).

In humans, iron deficiency leads to G1/S cell cycle arrest and to the induction of several members of the growth arrest and DNA damage (GADD) gene family members, probably associated with DNA damage resulting from iron deficiency (Saletta et al., 2011). In addition, DNA replication and repair depends on Fe-S clusters (Netz etal., 2010; Pokharel and Campbell, 2012) the synthesis of which is impaired upon iron deficiency (Chen etal., 2004). Defects in Fe-S cluster biogenesis result in genome instability (Veatch etal., 2009). Not surprisingly, mitochondrial dysfunction associated with compromised biogenesis of Fe-S clusters is tightly linked to iron homeostasis (Veatch et al., 2009).

DNA damage applies to the sites of the storage of genetic material, i.e., the nucleus, plastids, and mitochondria. More recently, nucleoli have been associated with a function of sensing DNA damage and as a storage facility for DNA repair proteins (Antoniali etal., 2013). In plants, iron is concentrated in the nuclei, particularly in nuclear substructures that were identified as the nucleoli by Perls/DAB and DAPI co-staining (Roschzttardtz et al., 2011). The nucleolus was described as a central hub for the coordination of stress responses such as DNA repair (Boulon et al., 2010; Antoniali et al., 2013), and for several DNA-repair-related proteins (Gao et al., 2003; Guo et al., 2008). While in plants such information is not available, we speculate that the high iron concentrations in nuclei/nucleoli are important for prioritizing DNA replication and repair when iron becomes limited.

\section{CONCLUSION}

In summary, the re-interpretation of the data outlined above hints at a new facet of the iron deficiency response of Arabidopsis, which might also be important for other plant species and may thus represent a general aspect in the adaptation of plants to low iron availability. Under iron shortage, DNA-related processes such as replication and repair might be prioritized to secure essential housekeeping functions. While alternative scenarios such as a role of UBC13 in root development that is not linked to DNA repair cannot be ruled out at present, the fact that $u b c 13 a$ mutants appear to have a higher, healthier iron status implies that activation of the DNA damage pathway occurs at the expense of other genes encoding iron-containing proteins. This situation is similar to what has been reported for yeast where iron deficiency limits iron utilization in energygenerating pathways via the action of the RNA-binding protein Cth2 to avoid that essential processes such as DNA synthesis and repair and thus genome integrity are compromised (Martínez-Pastor et al., 2013). While the evidence reported here is indirect and awaits experimental validation, it is tempting to assume that, similar to yeast, post-transcriptional mechanisms that bias the translation of messenger RNAs encoding proteins involved in essential iron-requiring processes such as DNA replication and repair also occur in Arabidopsis and other plants. Our initial experiments indicate that both UBC13 and RGLG are involved in DNA repair, but only for $u b c 13 a$ $u b c 13 b$ mutants iron deficiency severed the effect of MMS. This is consistent with a competition for UBC13 protein in the nucleus due to stress-induced translocation of RGLG1/2 to the nucleus. The role of RGLG1/2 under iron-deficient conditions awaits clarification, but from our results a function for RGLG1/2 in genome stability appears to be plausible. Further experiments addressing the fitness of plants treated with DNAdamaging agents and grown with different iron supply may falsify or validate our hypothesis. The effects associated with adverse environmental condition on genome stability are generally understudied and, despite big steps forward in the past few years (reviewed by Waterworth et al., 2011), not fully understood. From the example outlined here for iron deficiency it might be inferred that mechanisms that are involved in combating the constant assault of environmental stresses on genome stability may be an important component of the plant stress response.

\section{MATERIALS AND METHODS}

Arabidopsis thaliana (L.) Heynh Columbia (Col-0) ecotype was used as the wild-type. Seeds of the wild type, $u b c 13 a$ and $u b c 13 b$ mutants were obtained from the Arabidopsis Biological Resource Centre (Ohio State University), ubc13a ubc13b double mutants were generated by genetic crossing. The rglg1 rglg2 mutant lines were provided by Dr. Andreas Bachmeir (University of Vienna). Seeds were surface-sterilized and sown on media as described by Estelle and Somerville (1987), supplemented with 0\%, 0.006\%, 
$0.01 \%$, and $0.014 \%$ MMS either with or without $40 \mu \mathrm{M}$ FeEDTA, and stored at $4{ }^{\circ} \mathrm{C}$ for 2 days. A total of 36 seeds were placed on each plate, three plates were used for each treatment. After 5 days, seed germination was determined and normalized with the rate observed on iron-replete standard without MMS.

\section{ACKNOWLEDGMENTS}

We thank Thomas J. Buckhout (Humboldt University Berlin) for valuable suggestions and critical comments on the manuscript. Work in the Schmidt laboratory is supported by grants from Academia Sinica and the National Science Council.

\section{REFERENCES}

Antoniali, G., Lirussi, L., Poletto, M., and Tell, G. (2013). Emerging roles of the nucleolus in regulating the DNA damage response: the noncanonical DNA repair enzyme APE1/Ref-1 as a paradigmatical example. Antioxid. Redox Signal. doi:10.1089/ars.2013.5491

Benjamins, R., and Scheres, B. (2008). Auxin: the looping star in plant development. Annu. Rev. Plant Biol. 59, 443-465. doi: 10.1146/annurev. arplant.58.032806.103805

Boulon, S., Westman, B. J., Hutten, S., Boisvert, F. M., and Lamond, A. I. (2010). The nucleolus under stress. Mol. Cell 40, 216-227. doi: 10.1016/j.molcel.2010.09.024

Cavanaugh, P. F. Jr., Porter, C. W., Tukalo, D., Frankfurt, O. S., Pavelic, Z. P., and Bergeron, R. J. (1985). Characterization of L1210 cell growth inhibition by the bacterial iron chelators parabactin and compound II. Cancer Res. 45, 4754-4759.

Chang, D. J., and Cimprich, K. A. (2009). DNA damage tolerance: when it's OK to make mistakes. Nat. Chem. Biol. 5, 82-90. doi: 10.1038/nchembio.139

Chen, I. P., Mannuss, A., Orel, N., Heitzeberg, F., and Puchta, H. (2008). A homolog of ScRAD5 is involved in DNA repair and homologous recombination in Arabidopsis. Plant Physiol. 146, 1786-1796. doi: 10.1104/pp.108.116806

Chen, O. S., Crisp, R. J., Valachovic, M., Bard, M., Winge, D. R., and Kaplan, J. (2004). Transcription of the yeast iron regulon does not respond directly to iron but rather to iron-sulfur cluster biosynthesis. J. Biol. Chem. 279, 29513-29518. doi: 10.1074/jbc.M403209200

Cheng, M. C., Hsieh, E. J., Chen, J. H., Chen, H. Y., and Lin, T. P. (2012). Arabidopsis RGLG2, functioning as a RING E3 ligase, interacts with AtERF53 and negatively regulates the plant drought stress response. Plant Physiol. 158, 363-375. doi: $10.1104 / \mathrm{pp} .111 .189738$

Dinneny, J. R., Long, T. A., Wang, J. Y., Jung, J. W., Mace, D., Pointer, S., et al. (2008). Cell identity mediates the response of Arabidopsis roots to abiotic stress. Science 320, 942-945. doi: 10.1126/science. 1153795

Estelle, M. A., and Somerville, C. (1987). Auxin-resistant mutants of Arabidopsis thaliana with an altered morphology. Mol. Gen. Genet. 206, 200-206. doi: 10.1007/BF00333575

Fourcroy, P., Siso-Terraza, P., Sudre, D., Saviron, M., Reyt, G., Gaymard, F., et al. (2013). Involvement of the ABCG37 transporter in secretion of scopoletin and derivatives by Arabidopsis roots in response to iron deficiency. New Phytol. 201, 155-167. doi: 10.1111/nph.12471

Furukawa, T., Naitoh, Y., Kohno, H., Tokunaga, R., and Taketani, S. (1992). Iron deprivation decreases ribonucleotidereductase activity and DNA synthesis. Life Sci. 50, 2059-2065. doi: 10.1016/0024-3205(92)90572-7

Gao, H., Chen, X. B., and Mcgowan, C. H. (2003). Mus81 endonuclease localizes to nucleoli and to regions of DNA damage in human S-phase cells. Mol. Biol. Cell 14, 4826-4834. doi: 10.1091/mbc.E03-05-0276

Gollhofer, J., Schlawicke, C., Jungnick, N., Schmidt, W., and Buckhout, T. J. (2011). Members of a small family of nodulin-like genes are regulated under iron deficiency in roots of Arabidopsis thaliana. Plant Physiol. Biochem. 49, 557-564. doi: 10.1016/j.plaphy.2011.02.011

Grunewald, W., and Friml, J. (2010). The march of the PINs: developmental plasticity by dynamic polar targeting in plant cells. EMBO J. 29, 2700-2714. doi: 10.1038/emboj.2010.181

Guimil, S., and Dunand, C. (2007). Cell growth and differentiation in Arabidopsis epidermal cells. J. Exp. Bot. 58, 3829-3840. doi: 10.1093/jxb/erm253

Guo, Z., Qian, L., Liu, R., Dai, H., Zhou, M., Zheng, L., et al. (2008). Nucleolar localization and dynamic roles of flap endonuclease 1 in ribosomal DNA replication and damage repair. Mol. Cell. Biol. 28, 4310-4319. doi: 10.1128/MCB.00200-08
Hofmann, R. M., and Pickart, C. M. (1999). Noncanonical MMS2-encoded ubiquitin-conjugating enzyme functions in assembly of novel polyubiquitin chains for DNA repair. Cell 96, 645-653. doi: 10.1016/S0092-8674(00)80575-9

Jin, C. W., You, G. Y., He, Y. F., Tang, C. X., Wu, P., and Zheng, S. J. (2007). Iron deficiency-induced secretion of phenolics facilitates the reutilization of root apoplastic iron in red clover. Plant Physiol. 144, 278-285. doi: 10.1104/pp.107.095794

Lan, P., Li, W. F., Wen, T. N., and Schmidt, W. (2012). Quantitative phosphoproteome profiling of iron-deficient Arabidopsis roots. Plant Physiol. 159, 403-417. doi: 10.1104/pp.112.193987

Landsberg, E. C. (1996). Hormonal regulation of iron-stress response in sunflower roots: a morphological and cytological investigation. Protoplasma 194, 69-80. doi: 10.1007/BF01273169

Leitner, J., Retzer, K., Korbei, B., and Luschnig, C. (2012). Dynamics in PIN2 auxin carrier ubiquitylation in gravity-responding Arabidopsis roots. Plant Signal. Behav. 7, 1271-1273. doi: 10.4161/psb.21715

Li, W., and Schmidt, W. (2010). A lysine-63-linked ubiquitin chain-forming conjugase, UBC13, promotes the developmental responses to iron deficiency in Arabidopsis roots. Plant J. 62, 330-343. doi: 10.1111/j.1365-313X.2010. 04150.x

Ma, Z., Walk, T. C., Marcus, A., and Lynch, J. P. (2001). Morphological synergism in root hair length, density, initiation and geometry for phosphorus acquisition in Arabidopsis thaliana: a modeling approach. Plant Soil 236, 221-235. doi: 10.1023/A:1012728819326

Mannuss, A., Dukowic-Schulze, S., Suer, S., Hartung, F., Pacher, M., and Puchta, H. (2010). RAD5A, RECQ4A, and MUS81 have specific functions in homologous recombination and define different pathways of DNA repair in Arabidopsis thaliana. Plant Cell 22, 3318-3330. doi: 10.1105/tpc.110.078568

Martínez-Pastor, M. T., De Llanos, R., Romero, A. M., and Puig, S. (2013). Posttranscriptional regulation of iron homeostasis in Saccharomyces cerevisiae. Int. J. Mol. Sci. 14, 15785-15809. doi: 10.3390/ijms140815785

Müller, M., and Schmidt, W. (2004). Environmentally induced plasticity of root hair development in Arabidopsis. Plant Physiol. 134, 409-419. doi: 10.1104/pp.103.029066

Netz, D. J., Stumpfig, M., Dore, C., Muhlenhoff, U., Pierik, A. J., and Lill, R. (2010). Tah 18 transfers electrons to Dre2 in cytosolic iron-sulfur protein biogenesis. Nat. Chem. Biol. 6, 758-765. doi: 10.1038/nchembio.432

Pokharel, S., and Campbell, J. L. (2012). Cross talk between the nuclease and helicase activities of Dna2: role of an essential iron-sulfur cluster domain. Nucleic Acids Res. 40, 7821-7830. doi: 10.1093/nar/gks534

Rodríguez-Celma, J., Lin, W. D., Fu, G. M., Abadía, J., López-Míllan, A. F., and Schmidt, W. (2013). Mutually exclusive alterations in secondary metabolism are critical for the uptake of insoluble iron compounds by Arabidopsis and Medicago truncatula. Plant Physiol. 162, 1473-1485. doi: 10.1104/pp.113.220426

Roschzttardtz, H., Grillet, L., Isaure, M. P., Conejero, G., Ortega, R., Curie, C., et al. (2011). Plant cell nucleolus as a hot spot for iron. J. Biol. Chem. 286, 27863-27866. doi: 10.1074/jbc.C111.269720

Saletta, F., Suryo Rahmanto, Y., Siafakas, A. R., and Richardson, D. R. (2011). Cellular iron depletion and the mechanisms involved in the iron-dependent regulation of the growth arrest and DNA damage family of genes. J. Biol. Chem. 286, 3539635406. doi: 10.1074/jbc.M111.273060

Santi, S., Cesco, S., Varanini, Z., and Pinton, R. (2005). Two plasma membrane $\mathrm{H}^{(+)}$ATPase genes are differentially expressed in iron-deficient cucumber plants. Plant Physiol. Biochem. 43, 287-292. doi: 10.1016/j.plaphy.2005.02.007

Santi, S., and Schmidt, W. (2008). Laser microdissection-assisted analysis of the functional fate of iron deficiency-induced root hairs in cucumber. J. Exp. Bot. 59, 697-704. doi: 10.1093/jxb/erm351

Santi, S., and Schmidt, W. (2009). Dissecting iron deficiency-induced proton extrusion in Arabidopsis roots. New Phytol. 183, 1072-1084. doi: 10.1111/j.14698137.2009.02908.x

Sanvisens, N., Bano, M. C., Huang, M., and Puig, S. (2011). Regulation of ribonucleotidereductase in response to iron deficiency. Mol. Cell 44, 759-769. doi: 10.1016/j.molcel.2011.09.021

Savage, N., Yang, T. J., Chen, C. Y., Lin, K.-L., Monk, N. A. M., and Schmidt, W. (2013). Positional signaling and expression of ENHANCER OF TRY AND $\mathrm{CPC1}$ are tuned to increase root hair density in response to phosphate deficiency in Arabidopsis thaliana. PLoS ONE 8:e75452. doi:10.1371/journal.pone. 0075452 
Schikora, A., and Schmidt, W. (2001). Iron stress-induced changes in root epidermal cell fate are regulated independently from physiological responses to low iron availability. Plant Physiol. 125, 1679-1687. doi: 10.1104/pp.125. 4.1679

Schikora, A., and Schmidt, W. (2002). Formation of transfer cells and $\mathrm{H}^{(+)}$-ATPase expression in tomato roots under P and Fe deficiency. Planta 215, 304-311. doi: 10.1007/s00425-002-0738-0

Schmidt, W., and Bartels, M. (1996). Formation of root epidermal transfer cells in Plantago. Plant Physiol. 110, 217-225.

Schmidt, W., Michalke, W., and Schikora, A. (2003). Proton pumping by tomato roots. Effect of Fe deficiency and hormones on the activity and distribution of plasma membrane $\mathrm{H}^{+}$-ATPase in rhizodermal cells. Plant Cell Environ. 26 361-370. doi: 10.1046/j.1365-3040.2003.00967.x

Susín, S., Abian, J., Sanchez-Baeza, F., Peleato, M. L., Abadía, A., Gelpi, E., et al. (1993). Riboflavin $3^{\prime}$ - and $5^{\prime}$ sulfate, two novel flavins accumulating in the roots of iron-deficient sugar beet (Beta vulgaris). J. Biol. Chem. 268, 2095820965.

Thimm, O., Essigmann, B., Kloska, S., Altmann, T., and Buckhout, T. J. (2001) Response of Arabidopsis to iron deficiency stress as revealed by microarray analysis. Plant Physiol. 127, 1030-1043. doi: 10.1104/pp.010191

Ticconi, C. A., Lucero, R. D., Sakhonwasee, S., Adamson, A. W., Creff, A., Nussaume, L., et al. (2009). ER-resident proteins PDR2 and LPR1 mediate the developmental response of root meristems to phosphate availability. Proc. Natl. Acad. Sci. U.S.A 106, 14174-14179. doi: 10.1073/pnas.0901778106

Ulrich, H. D. (2007). Conservation of DNA damage tolerance pathways from yeast to humans. Biochem. Soc. Trans. 35, 1334-1337. doi: 10.1042/BST0 351334

VanDemark, A. P., Hofmann, R. M., Tsui, C., Pickart, C. M., and Wolberger, C. (2001). Molecular insights into polyubiquitin chain assembly: crystal structure of the Mms2/Ubc13 heterodimer. Cell 105, 711-720. doi: 10.1016/S00928674(01)00387-7

Veatch, J. R., McMurray, M. A., Nelson, Z. W., and Gottschling, D. E. (2009). Mitochondrial dysfunction leads to nuclear genome instability via an iron-sulfur cluster defect. Cell 137, 1247-1258. doi: 10.1016/j.cell.2009.04.014

Vicente-Agullo, F., Rigas, S., Desbrosses, G., Dolan, L., Hatzopoulos, P., and Grabov, A. (2004). Potassium carrier TRH1 is required for auxin transport in Arabidopsis roots. Plant J. 40, 523-535. doi: 10.1111/j.1365-313X.2004.02230.x
Wang, S., Wen, R., Shi, X., Lambrecht, A., Wang, H., and Xiao, W. (2011). RAD5a and REV3 function in two alternative pathways of DNA-damage tolerance in Arabidopsis. DNA Repair 10, 620-628. doi: 10.1016/j.dnarep.2011.04.009

Waterworth, W. M., Drury, G. E., Bray, C. M., and West, C. E. (2011). Repairing breaks in the plant genome: the importance of keeping it together. New Phytol. 192, 805-822. doi: 10.1111/j.1469-8137.2011.03926.x

Wen, R., Newton, L., Li, G. Y., Wang, H., and Xiao, W. (2006). Arabidopsis thaliana UBC13: implication of error-free DNA damage tolerance and Lys63-linked polyubiquitylation in plants. Plant Mol. Biol. 61, 241-253. doi: 10.1007/s11103-006-0007-x

Wen, R., Torres-Acosta, J. A., Pastushok, L., Lai, X., Pelzer, L., Wang, H., et al. (2008) Arabidopsis UEV1D promotes lysine-63-linked polyubiquitination and is involved in DNA damage response. Plant Cell 20, 213-227. doi: 10.1105/tpc.107.051862

Wu, X. F., Yamamoto, M., Akira, S., and Sun, S. C. (2009). Regulation of hematopoiesis by the K63-specific ubiquitin-conjugating enzyme Ubc13. Proc. Natl. Acad. Sci. U.S.A. 106, 20836-20841. doi: 10.1073/pnas.0906547106

Yin, X. J., Volk, S., Ljung, K., Mehlmer, N., Dolezal, K., Ditengou, F., et al. (2007). Ubiquitin lysine 63 chain forming ligases regulate apical dominance in Arabidopsis. Plant Cell 19, 1898-1911. doi: 10.1105/tpc.107.052035

Conflict of Interest Statement: The authors declare that the research was conducted in the absence of any commercial or financial relationships that could be construed as a potential conflict of interest.

Received: 11 October 2013; accepted: 12 December 2013; published online: 02 January 2014.

Citation: Pan I-C and Schmidt W (2014) Functional implications of K63-linked ubiquitination in the iron deficiency response of Arabidopsis roots. Front. Plant Sci. 4:542. doi: 10.3389/fpls.2013.00542

This article was submitted to Plant Genetics and Genomics, a section of the journal Frontiers in Plant Science.

Copyright (c) 2014 Pan and Schmidt. This is an open-access article distributed under the terms of the Creative Commons Attribution License (CC BY). The use, distribution or reproduction in other forums is permitted, provided the original author(s) or licensor are credited and that the original publication in this journal is cited, in accordance with accepted academic practice. No use, distribution or reproduction is permitted which does not comply with these terms. 